\title{
Genetic susceptibility to oral cancer by human papillomavirus infection
}

\section{Martha Rebolledo-Cobos ${ }^{1 *}$ and Zuleima Yáñez-Torregroza ${ }^{2}$}

${ }^{1}$ Universidad Metropolitana, Dentistry Program, Atlántico, Colombia; ${ }^{2}$ Universidad Simón Bolívar, Faculty of Medicine, Basic and Biomedical Sciences, Atlántico, Colombia

\begin{abstract}
Environmental carcinogens and human papillomavirus (HPV) are the main responsible factors for oral cancer. Susceptibility factors in the human genome play a risk-modulating role; however not all individuals exposed to these carcinogens suffer from cancer. The purpose of the present review is to describe the main factors of genetic susceptibility to oral cancer due to HPV infection. A systematic search was carried out in three databases in English, with only 7 articles meeting the selection criteria. Genetic polymorphisms are shown in three categories, which are related to HPV and participate in oncogenesis. Three articles related to deregulation of cell cycle control mechanisms were identified, as well as one referring to mutations in the apoptosis pathway and three about polymorphisms in inflammatory and immune response genes. The association of polymorphisms for the development of oral cancer by HPV is evident, although it remains under study. Oral neoplasms' oncogenesis pattern is not always associated with HPV, but with other environmental or epigenetic factors.
\end{abstract}

KEY WORDS: Genetic susceptibility. Oral cancer. Polymorphisms. Human papillomavirus. Mutation.

\section{Introduction}

The description of the variants in human DNA sequences and protein sequences started with two independent publications by Beaudet et al. and Beutler et al. in 1993. Those authors proposed that any abnormal change in the nucleotide sequence is usually a mutation, ${ }^{1,2}$ which may or may not cause phenotypic changes in affected individuals and not necessarily corresponds to a disease and can be inherited from the parents (germline mutation) or acquired and manifest itself throughout an individual's life (somatic or multifactorial mutation).

In the latter mutation type, there is specifically a variation in the DNA sequence that can occur in a population with a frequency of $1 \%$ or higher, and that is known as polymorphism. ${ }^{3}$ Polymorphisms occur naturally, with a neutral effect, beneficial or promoter of disease. They can also involve one or more nucleotide changes, as well as other mutations. Single nucleotide polymorphism is the most common, and it arises from every 1000 base pairs (bp) in the human genome and is usually found in areas that flank protein encoding genes or in regions recognized as critical for microRNA binding and gene/protein expression regulation. ${ }^{3}$ However, single nucleotide polymorphisms can also occur in coding sequences, introns or intergenic regions. ${ }^{4}$

In the context of hereditary and multifactorial diseases, a mutation has been defined as an alteration 
in the gene sequence that nullifies its biological function; it is also possible to find it in malignant tumors of the oral cavity when the individual is exposed to certain viruses or epigenetic risk-factors. ${ }^{5}$ In general, there are few types of cancer where one or more genes whose mutations confer a high risk for developing them have been able to be identified. As for oral cancer (OC), especially squamous cell carcinoma and other head and neck cancers, although it is true that they have multiple etiologies and acquired risk factors such as alcohol consumption or smoking, sexually transmitted diseases or infections with human immunodeficiency, herpes simplex or human papilloma virus (HPV) play an important role. ${ }^{6,7}$ In the latter case, mutations of different kinds are identified, which confer susceptibility to neoplastic diseases originating in a primary viral infection at some point in life..$^{4-7} \mathrm{HPV}$ is the cause of other neoplastic lesions that sometimes persist, but not necessarily evolve into cancer, which is related to its genotypes: high risk (hrHPV) and low risk (IrVHP); the most commonly associated with $O C$ are genotypes $16,18,31$ and 45 , considered to be of high risk. ${ }^{6-8}$

There are investigations that associate the somatic component with the appearance of hrHPV-attributed $O C$ due to changes in the genes involved with the cell cycle (which play a role in the modulation of cell DNA repair), control of the cell cycle, cell growth, apoptosis and inflammatory and immune response, which in physiological conditions usually provide stability to the human genome..$^{8-14}$ This systematic review has as its goal to describe the main factors of genetic susceptibility for the development of OC or squamous cell carcinoma in association with HPV infection.

\section{Method}

- Population: Patients infected with HPV and who developed OC.

- Interventions: Molecular diagnosis of mutations and HPV genotyping with regard to the presence of OC.

- Objective: To identify susceptibility factors for OC associated with HPV infection.

- Included studies: case-control studies, systematic reviews or meta-analyses.

\section{Inclusion criteria}

- Full-text articles written in English.

- Studies published between January 1, 2013 and January 31, 2018.
- Studies assessing genetic susceptibility to OC due to HPV infection.

- Studies provided information on cell cycle control polymorphisms in OC associated with HPV.

- Studies that provided information on polymorphisms by variations in apoptosis.

- Studies that would provide information on polymorphisms of inflammatory and immune response genes.

\section{Exclusion criteria}

- Studies that provided inadequate information.

- Narrative reviews.

- In vitro studies.

- Animal studies.

- Studies involving potentially malignant alterations.

- Studies that included other infections, such as human immunodeficiency virus or herpes simplex.

- Studies where no molecular diagnosis and genotyping had been performed.

\section{Sources or information and search}

An extensive systematic literature search was manually conducted in the PubMed, Elsevier and Clinical Key databases, using the combination of the following descriptors: HPV and oral cancer, human susceptibility, HPV infection, oral cavity, polymorphisms of a single nucleotide, polymorphisms of cell cycle control, variations in apoptosis and polymorphisms of inflammatory and immune response genes. The articles involved only investigations in humans, with no gender or age distinction (Figure 1).

\section{Study selection}

Initially, 115 articles were recorded in an electronic spreadsheet. After eliminating duplicate articles, only 64 were considered relevant due to their titles and abstracts; 18 were identified to assessed susceptibility to HPV infection in the oral cavity, out of which 11 were excluded because they failed to meet the selection criteria. Only seven articles were included in the final analysis (Figure 1).

\section{Data collection}

A standard pilot-type instrument was provided for data extraction by the expert; only the appropriate elements were taken into account for this review. The 
- 115 articles were initially identified in the PubMed, Elsevier and Clinical Key databases

- 11 were excluded because they did not meet the selection criteria: 4 did not comply with the publication dates, 3 were clinical cases, 2 were narrative reviews and 2 involved the Eligibility presence of other viruses

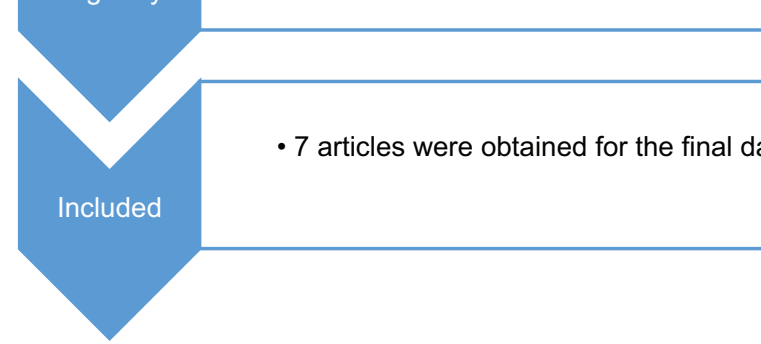

Figure 1. Flowchart of the selection process of reviewed articles.

expert verified and approved the data, which were extracted and tabulated in an electronic Excel spreadsheet, one for each selected article.

Of the seven articles, three referred to polymorphisms of the cell cycle control, one to polymorphisms due to apoptosis variations and three to polymorphisms of inflammatory and immune response genes (Figure 1).

The data of interest were the following: name of the article, author name, country, publication year, study design, sample size, composition, population, genotyping, polymorphism/mutation type, type of cancer, presence of HPV infection, risk factors or comparison intervention, risk level, results and observations.

\section{Results}

For hrHPV infection to persist and extrapolate to cancer development, there are host genetic factors that contribute to the variability these malignant neoplasms develop with after viral infection. ${ }^{7} \mathrm{HPV}$ viral oncoproteins E6 and E7 deactivate tumor suppressor genes p53 and pRb, which allows the cell to escape the normal cell cycle checkpoints, with subsequent cell transformation, immortalization and oncogenesis ${ }^{7}$ Several authors point at three simultaneous pathways for genetic polymorphisms analysis that can help understand the different mechanisms involved in the appearance of HPV-associated OC and squamous cell carcinoma: cell cycle control polymorphisms, apoptosis variations and inflammatory and immune response genes polymorphisms. ${ }^{7}$

\section{Cell cycle control polymorphisms}

The first type of polymorphism corresponds to the interactions between the human genome and the $\mathrm{hrH}$ PV genome; the mechanism of action lies in the relationship between tumor suppressor gene p53 and HPV oncoprotein E6. This non-structural protein binds to the p53 gene and starts its degradation, which causes uncontrolled cell proliferation. ${ }^{7,8}$ Gene p53 is located on the short arm of chromosome 17, band 13 (17p13.1), has approximately $20 \mathrm{~kb}$ and consists of 11 exons, the first one non-coding and placed between 8 and $10 \mathrm{~kb}$ of exons 2 to 11 . Ninety percent of this type of mutations have been located in exons 5 to 8 of the gene; approximately 20 to $30 \%$ of mutations occur in all five hot-spot codons found in these exons.

Other p53 alterations are deletions, insertions, mutations in splicing sites and heterozygosity losses. ${ }^{7,8}$ The most common mechanism of p53 functionality loss is point mutation of one of the alleles and deletion of the other. In this sense, it should be noted that some p53 mutations are dominant, which is an 
exception to the rule that suppressor genes manifest their oncogenic action only if there is an alteration of both gene copies;:910 a single defective protein monomer stemming from the mutation of one of the two alleles of the p53 gene is enough to cause total inactivation of the tetrameric protein. When mutated monomers form complexes with normal monomers, a mutant p53 protein with noticeably lengthened a halflife is formed. ${ }^{7.8}$

\section{Apoptosis variations}

During carcinogenesis, tumors have to develop multiple mechanisms to overcome host immune surveillance and intrinsic apoptosis or cell cycle arrest. The individual difference in resistance to apoptosis through the FAS pathway might allow many cancers to escape the immune system or counterattacking it. The germline variants in extrinsic and intrinsic pathways might affect apoptotic efficacy and resistance to apoptosis and, consequently, influence on HPV infection. ${ }^{7,11}$ This can be particularly relevant to a viral mechanism that works through the cell cycle and apoptotic mechanisms. Gene polymorphisms of the FAS and FASL promoters have been suggested to contribute to the risk of HPV-associated cancer by inducing immune cells differential apoptosis in response to micro-environmental signals after HPV infection. The polymorphism at position 670 of the FAS promoter has been found to suppress the binding site for the transcription nuclear element and to alter the expression of the FAS gene; pro-72 homozygote $p 53$ polymorphism in codon 72 appears to be an important apoptosis regulator through the FAS/FASL pathway in head and neck cancer. ${ }^{11}$

\section{Inflammatory and immune response gene polymorphisms}

While hrHPV are well understood as risk factors for $\mathrm{OC}$ and head and neck cancer, research on host genetic factors in inflammatory and immune responses to HPV infection might help understanding the association between HPV infection and OC. It is precisely these responses or immune surveillance efficacy that modifies HPV elimination or persistent infection. ${ }^{7,12}$ Inflammation is part of the host response to internal or external environmental stimuli, which is promoted by the action of pro-inflammatory cytokines, including interleukins (IL) 1 and 6, tumor necrosis factor and interferon- $\gamma$, and is commonly resolved with anti-inflammatory agents. Cytokines IL-4, IL-10, IL-13, as well as interferon- $\alpha$ and transforming growth factor- $\beta$ play a role in the control of HPV-infected cell growth. Viral persistence, disease progression or transformation into cancer involve escaping from these mechanisms. Therefore, germline variants of these cytokines might modify the effectiveness of the defense against HPV and, consequently, infection rates. ${ }^{12}$

The success of HPV in decreasing immune responses may be important in the pathogenesis of HPV-associated cancer, since the etiology of HPV-induced cancer triggers a persistent viral infection that can be minimized by an effective immune response. The polymorphisms of several cytokine genes have been implicated in an induction of susceptibility or resistance to cancers caused by HPV infection due to their role in determining the host immune response. Therefore, genetic variants of cytokine genes in promoter or coding regions that are thought to influence on expression levels or functional efficacy may be involved in susceptibility to the HPV status of patients with squamous cell carcinoma and OC.13,14

Some studies have associated polymorphisms in pro-inflammatory and anti-inflammatory cytokine genes, particularly in regulatory regions, with intra-individual variations in cytokine production and cancer risk. The association of cytokines interferon- $\gamma$ and IL10 polymorphisms with cancer risk has been well documented: interferon- $\gamma$ decreased transcription and IL10 increased transcription has been observed in patients with HPV-positive cervical cancer..$^{12,14}$ Interferon- $\gamma$ plays an essential role in the defense against intracellular viruses and pathogens by inducing immune-mediated inflammatory responses. Single nucleotide polymorphism $\mathrm{T}+874 \mathrm{~A}$, located at interferon- $\gamma$ gene translation start site, which coincides with a binding site for the putative nuclear factor-kappa B transcription factor, could be fundamental in the induction of a constitutively high interferon- $\gamma$ production. The $+874 \mathrm{~T}$ to $\mathrm{A}$ alleles with low $(\mathrm{AA})$, medium (AT) and high (TT), have also been significantly associated with cytokine production. IL-10 has a suppressive effect on cell-mediated immunity, which can be critical in eliminating QTs that host HPVg. ${ }^{13}$ There are different polymorphisms in the IL-10 gene, among which that of a single nucleotide in position 1082 of the promoter region plays an important role in determining high, medium and low production of IL-10.

The association of $\mathrm{G} / \mathrm{A}$ single nucleotide polymorphism in position 1082 has been associated with low 
Table 1. Cell cycle control polymorphisms related to human papillomavirus in oral cancer and squamous cell carcinoma

\begin{tabular}{|c|c|c|c|c|c|c|c|}
\hline Polymorphism & Mutation & Genotype frequency & $n$ & $p$ & Oncologic diagnosis & Country & Author \\
\hline \multirow[t]{3}{*}{$\begin{array}{l}\text { Cell cycle } \\
\text { control pathway }\end{array}$} & p53 codon 72 & $\begin{array}{l}\text { Arg/Arg }=23 \%(8) \\
\text { Arg/Pro }=57 \%(20) \\
\text { Pro/Pro }=20 \%(7)\end{array}$ & 35 & $<0.809$ & SCC & India & Tandon et al. ${ }^{8}$ \\
\hline & p53 codon 72 & $\begin{array}{l}\text { Arg }) / \operatorname{Arg}=8.47 \%(22) \\
\text { Arg/Pro }=43.46 \%(113) \\
\text { Pro/Pro }=4807 \%(125)\end{array}$ & 260 & $<0.001$ & SCC & Pakistan & Saleem et al. ${ }^{9}$ \\
\hline & p27 & $\begin{array}{l}\text { p27 (rs34329; } 3.05 \text { ratio, } \\
\text { 95\% Cl = 2.12-4.40), cyclin } \\
\text { E (rs1406), cyclin H (rd3093816), } \\
\text { cyclin D1-1 (rs647451), cyclin } \\
\text { D2 (rs3217901) and Rb } \\
\text { 1-2 (rs3092904). }\end{array}$ & $6 / 9$ & $<0.0001$ & OC & India & Murali et al. ${ }^{10}$ \\
\hline
\end{tabular}

$A A$, medium $A G$ and high $G G$ cytokines production. Polymorphisms in human IL-1b and tumor necrosis factor- $\alpha$ genes have also been reported to influence on cytokine and transforming growth factor expression, which regulates epithelial cell proliferation and apoptosis., 7,13,14 Tumor necrosis factor- $\alpha$ can directly control HPV infection by apoptosis induction in HPV-infected cells, such as cervical cancer and OC cells. Disturbance of the balance between pro and anti-inflammatory cytokine levels may be caused by inherited genetic mutations, from which common genetic variants can also modify key genes expression or function, disrupting cytokine balance and affecting cancer risk and outcome. . $^{13,14}$

The investigations examined for the synthesis of this review show biotransformation, detoxification, elimination or immune control of carcinogens such as HPV, together with cell regulatory mechanisms, DNA repair and apoptotic pathways, as intrinsic strategies to evade cancer. ${ }^{7}$ Tandon et al. and Saleen et al. describe polymorphisms in the $p 53$ gene located on the short arm of chromosome 17, band 13 (17p13.1). In turn, Murali et al. associate them with the $p 27$ gene and consider that they contribute to $\mathrm{OC}$ pathogenesis. Only three articles related to this type of polymorphism were found, two from India and one from Pakistan ${ }^{10}$ (Table 1).

Only one article showed evidence that there are mechanisms to overcome host immune surveillance and intrinsic apoptosis. Sun et al. consider that the FAS/FASL promoter variants in the apoptosis pathways alter the transcriptional activity of those genes and cell death regulation. However, they state that no study has investigated if tumor sites contribute to the association between the FAS/FASL polymorphisms and the risk of tumor recurrence. ${ }^{11}$ They also identified that patients with genotype index for oropharyngeal
Table 2. Apoptosis pathway polymorphisms ${ }^{11}$

\begin{tabular}{|c|l|c|c|}
\hline Pathway & Genotype & n & $\%$ \\
\hline $\begin{array}{c}\text { FAS (mutación) } \\
\text { FAS 670 A > G }\end{array}$ & AA & 7 & 16.7 \\
\hline FAS 1377 G > A & AG + GG & 35 & 83.3 \\
\hline FASL (mutación) & AG +AA & 34 & 80.9 \\
\hline FASL 124 A > G & AA & & 19.1 \\
\hline FASL 844 C > T & AG + GG & 31 & \\
\hline (mutación = mutation) & CC & 11 & 26.2 \\
\hline & & 11 & 26.2 \\
\hline
\end{tabular}

cancer and FASL 844 CT/TT had a significantly higher risk of cancer recurrence $(\mathrm{cHR}=2.5,95 \%$ $\mathrm{Cl}=1.1-5.8, \mathrm{p}=0.043 ; \mathrm{HR}=2.7,95 \% \mathrm{Cl}=1.2-6.0$, $\mathrm{p}=0.032$ ) in comparison with patients with the FASL 844 CC genotype as the reference group, while patients without oropharyngeal cancer with the FAS 670 AG/GG and FASL 844 CT/TT genotypes had a significantly higher risk than those patients at risk of tumor recurrence ( $\mathrm{cHR}=2.2$ and $1.8,95 \% \mathrm{Cl}=1.2-5.7$ and 1.1-3.2, $\mathrm{p}=0.04$ and 0.041 , respectively; $\mathrm{aHR}=2.4$ and $1.7,95 \% \mathrm{Cl}=1.1-5.1$ and $1.0-3.0, \mathrm{p}=0.043$ and 0.049 , respectively) in comparison with their corresponding AA and CC genotypes ${ }^{11}$ (Table 2).

In the examined investigations, hrHPVs were widely described as risk factors for head and neck cancer and OC. The host produces inefficacious immune surveillance responses against HPV infections, which causes for viral presence and malignant transformation to perpetuate; three articles were found in this regard.

Jin et al. found that HPV 16 seropositivity was only associated with an increased risk of OC (OR $=3.1,95$ $\% \mathrm{Cl}=2.1-4.6)$ and that the risk of oropharyngeal cancer associated with HPV 16 was modified for each 
Table 3. Inflammatory and immune response genes' polymorphisms

\begin{tabular}{|c|c|c|c|c|c|c|}
\hline Polymorphism & $\mathrm{n}$ & Risk factor & $\begin{array}{l}\text { HPV } \\
\text { genotype }\end{array}$ & Risk & Cancer & Author \\
\hline $\begin{array}{l}\text { Single nucleotide polymorphism } \\
\text { TNF- } \alpha \\
{[-308 G>A(\text { rs1800629), }} \\
-857 C>\text { T (rs1799724), } \\
-863 C>A(r s 1800630) \text { y } \\
-1031 T>C(\text { rs1799964)] }\end{array}$ & $176 / 325$ & $\begin{array}{l}\text { - Tobacco consumption } \\
\text { - Alcohol consumption } \\
\text { - Age } \\
\text { - Gender }\end{array}$ & 16 hrHPV & $\begin{aligned} \mathrm{OR} & =3.1 \\
95 \% \mathrm{Cl} & =2.1-4.6\end{aligned}$ & HNC & Jin et al..$^{12}$ \\
\hline $\begin{array}{l}\text { TGF-b1 (G to C } \\
\text { polymorphism in codon } 25 \\
<\text { p915> and IL-10 (1082 G/A), } \\
-819 \mathrm{C} / \mathrm{T} \text { and } 592 \mathrm{C} / \mathrm{A})\end{array}$ & 161 & $\begin{array}{l}\text { - Tobacco consumption } \\
\text { - Alcohol consumption } \\
\text { - Age } \\
\text { - Gender } \\
\text { - Tumor classification } \\
\text { and localization }\end{array}$ & HVP+ & $\begin{array}{c}\mathrm{OR}=11.09 \\
95 \% \mathrm{Cl}=6.16-113.23\end{array}$ & $\mathrm{OC}$ & Hsu et al. ${ }^{13}$ \\
\hline $\begin{array}{l}\text { Single-nucleotide polymorphism } \\
\text { EVER } 1 \text { (rs2613516, rs12449858) } \\
\text { and EVER } \\
2 \text { (rs7205422, rs12452890) }\end{array}$ & $0 / 219$ & None & HVP + & & HNC & Antonsson et al. ${ }^{14}$ \\
\hline
\end{tabular}

single nucleotide polymorphism. ${ }^{12}$ In addition, similar results were observed for combined-risk genotypes of four variants, and all these significant associations were more pronounced in several subgroups, particularly in patients with oropharyngeal cancer that never smoked. ${ }^{12}$

In turn, Hsu et al. stated that there are two cytokines involved in carcinogenesis. ${ }^{13}$ They observed an association between the polymorphism in TGF-b1 ( $G$ to $C$ polymorphism at codon $25<+915>$ ), IL-10 (1082 G/A, $819 \mathrm{C} / \mathrm{T}$ and $592 \mathrm{C} / \mathrm{A}$ ) and $\mathrm{OC}$ risk in 162 patients They found that the TGF-b1 genotype 25 GC codon is significantly more common in patients with $\mathrm{OC}$ in comparison with a healthy control a group $(p<0.0001)$. Patients with genotype GC at codon 25 had an 11.09fold higher risk of OC $(\mathrm{OR}=11.09,95 \% \mathrm{Cl}=6.16$ 113.23). They demonstrated that IL-10 polymorphisms in positions 819 and 592 were correlated with OC risk $(p<0.0001)$. The genotypes that exhibited IL-10-592 $\mathrm{C}$ alleles had a higher risk of $\mathrm{OC}(\mathrm{OR}=1.79,95 \% \mathrm{Cl}$ $=1.11-2.91)$. Patients with the TC genotype in IL-10819 had 3.32 higher risk of OC (OR $=3.32,95 \% \mathrm{Cl}$ $=1.64-6.94) \cdot{ }^{13}$

Antonsson et al. suggest that polymorphisms in the two EV genes (EVER1 and EVER2, also known as transmembrane channel 6 and 8 proteins) are involved, since a small number of critical mutations in these genes cause HPV infections and cancer development. These authors failed to show evidence of any association with the state of single nucleotide polymorphisms EVER1/EVER2 and the HPV status, or with the presence of $\mathrm{OC}^{14}$ (Table 3).

\section{Conclusions}

The studies examined in this review show ample evidence of the relationship between HPV infection and the presence of $\mathrm{OC}$, apart from epigenetic risk factors, such as tobacco and alcohol consumption, age and modified sexual intercourse. Viral integration in the cell genome of the oral mucosa makes the human being genetically susceptible to the development of malignant tumors. The presence of three categories of genetic polymorphisms and mechanisms of simultaneous participation has been observed in the oncogenesis process, as well as the identification of transforming genes or oncoproteins that induce deregulation of the cell cycle control mechanisms, thus producing instability in the genome, apoptosis alteration and instability in inflammatory and immune response genes.

The study of the association of these polymorphisms with the development of $\mathrm{OC}$ in association with HPV infection is still under development; in addition, the oncogenesis pattern of all of head and neck cancers or $\mathrm{OC}$ is not always exclusively related to the presence of HPV, but with different environmental or hereditary risk factors. In the Colombian context, no exhaustive studies were found emphasizing the susceptibility of human genetics to $\mathrm{OC}$, squamous cell carcinoma or head and neck cancer associated with HPV infection.

\section{References}

1. Beaudet AL, Tsui LC. A suggested nomenclature for designating mutations. Hum Mutat. 1993;2:245-248. 
2. Beutler E. The designation of mutations. Am J Hum Genet. 1993;53:783-785

3. Karki R, Pandya D, Elston RC, Ferlini C. Defining "mutation" and "polymorphism" in the era of personal genomics. BMC Med Genomics. 2015;15;8:37.

4. Lacko M, Braakhuis BJ, Sturgis EM, Boedeker CC, Suárez C, Rinaldo A, et al. Genetic susceptibility to head and neck squamous cell carcinoma. Int J Radiat Oncol Biol Phys. 2014:89:38-48.

5. Hino O, Kobayashi T. Mourning Dr. Alfred G. Knudson: the two-hit hypothesis, tumor suppressor genes, and the tuberous sclerosis complex Cancer Sci. 2017;108:5-11.

6. Famooto A, Almujtaba M, Dareng E, Akarolo-Anthony S, Ogbonna C, Offiong R, et al. RPS19 and TYMS SNPs and Prevalent high risk human papilloma virus infection in Nigerian women. PLoS One. 2013:27;8:e66930.

7. Kenney AD, Dowdle JA, Bozzacco L, McMichael TM, St Gelais C, Panfil AR, et al. Human genetic determinants of viral diseases. Annu Rev Genet. 2017;51:241-263.

8. Tandon N, Srivastava AN, Fatima N, Raza ST, Kumar V. p53 codon 72 gene polymorphism studies and p53 expression by immunohistochemistry in oral lesions as risk factor for malignancy. Int J Appl Basic Med Res. 2017;7:243-246.
9. Saleem S, Azhar A, Hameed A, Khan MA, Abbasi ZA, Qureshi NR, et al. P53 (Pro72Arg) polymorphism associated with the risk of oral squamous cell carcinoma in gutka, niswar and manpuri addicted patients of Pakistan. Oral Oncol. 2013;49:818-823.

10. Murali A, Nalinakumari KR, Thomas S, Kannan S. Association of single nucleotide polymorphisms in cell cycle regulatory genes with oral cancer susceptibility. Br J Oral Maxillofac Surg. 2014;52:652-658.

11. Sun Y, Yu W, Sturgis EM, Peng W, Lei D, Song X, et al. Site disparities in apoptotic variants as predictors of risk for second primary malignancy in patients with squamous cell carcinoma of the head and neck. BMC Cancer. 2016;16:70

12. Jin L, Sturgis EM, Zhang Y, Huang Z, Song X, Li C, et al. Association of tumor necrosis factor-alpha promoter variants with risk of HPV-associated oral squamous cell carcinoma. Mol Cancer. 2013;12:80.

13. Hsu HJ, Yang YH, Shieh TY, Chen $\mathrm{CH}$, Kao YH, Yang CF, et al. TGF-b1 and IL-10 single nucleotide polymorphisms as risk factors for oral cancer in Taiwanese. Kaohsiung J Med Sci. 2015;31:123-129.

14. Antonsson A, Law MH, Neale RE, Coman WB, Pryor DI, Whiteman DC, et al. Variants of EVER1 and EVER2 (TMC6 and TMC8) and human papillomavirus status in patients with mucosal squamous cell carcinoma of the head and neck. Cancer Causes Control. 2016;27:809-815.g 